\title{
Enhanced Vascular Reactivity to Norepinephrine in Salt-Sensitive Patients with Hypertension
}

\author{
Rieko Shikuma, M.D., Manabu Yoshimura, M.D., \\ Hiroshi Ashizawa, M.D., Yoshihiro Kajita, M.D., \\ Hakuo Takahashi, M.D., Kazuo Takeda, M.D., \\ and Hamao IJIGHI, M.D.
}

\begin{abstract}
Summary
Twenty patients with hypertension were studied under diets containing low and high salt to identify factors which might be involved in elevating blood pressure under sodium-loading. They were classified as "salt-sensitive" (SS) and "nonsalt-sensitive" (NSS) according to the presence or absence of greater than $10 \%$ increases in mean blood pressure when a low salt diet was replaced by a high salt diet. During high-sodium intake, the SS patients showed reduced urinary excretion of sodium and elevated plasma levels of aldosterone as compared with plasma renin activity. The SS patients also showed an enhanced pressor response to norepinephrine under both low-sodium and high-sodium diets.

From these results, it is suggested that the sodium retention, which is probably related to nonsuppressed levels of PAC under sodium-loading, is one of the factors in elevating blood pressure in the SS patients. Moreover, the enhanced pressor response to norepinephrine seems to contribute, in part, to elevation of blood pressure in the SS patients under salt-loading.
\end{abstract}

\section{Additional Indexing Words:}

Salt-sensitive hypertension Nonsalt-sensitive hypertension Pressor response Norepinephrine Renin-aldosterone

$7 \mathrm{HE}$ major effects of dietary salt on incidence of hypertension ${ }^{1)}$ and development of hypertension ${ }^{2)}$ have been investigated in a number of epidemiological and experimental studies. It has been proposed that the expansion of the extracellular fluid volume by salt-loading is an important event in the elevation of blood pressure and the development of hypertension. ${ }^{3)}$ HowKyoto.

From the Second Department of Internal Medicine, Kyoto Prefectural University of Medicine,

Address for reprint: Manabu Yoshimura, M. D., Second Department of Internal Medicine, Kyoto Prefectural University of Medicine, Kawaramachi-hirokoji, Kamigyo-ku, Kyoto 602, Japan.

Received for publication December 24, 1981.

Manuscript revised May 10, 1982. 
ever, since salt-loading does not play a significant role in elevating blood pressure in normal subjects, other factors may contribute to the elevation of blood pressure. One of these factors is probably of genetic origin. Dahl et al4) demonstrated that the blood pressure in one strain of rats increases significantly with dietary salt intake (salt-sensitive rat), while the blood pressure in another strain is not affected by salt intake (salt-resistant rat).

In clinical studies, it was reported that patients with idiopathic hypertension also showed different circulatory responses to salt intake and were classified into salt-sensitive (SS) or nonsalt-sensitive (NSS) groups, as estimated from the elevation in blood pressure with increases in salt intake. ${ }^{5 /, 6)}$ From these investigations on a high-salt diet, the SS hypertensive patients displayed greater increases in blood pressure, more weight gain, more sodium retention ${ }^{5)}$ and greater increases in cardiac output ${ }^{6)}$ than NSS patients. Therefore, it is interesting to analyse the mechanisms involved in elevating blood pressure or retaining sodium in the SS hypertensive patients as compared with the NSS hypertensive patients. In the present study, we investigated the vascular reactivity to exogenous norepinephrine and the responses of plasma renin activity (PRA) and plasma aldosterone concentration (PAG) to salt-loading, and attempted to estimate the factors involved in inducing salt-sensitive hypertension.

\section{Subjects and Methods}

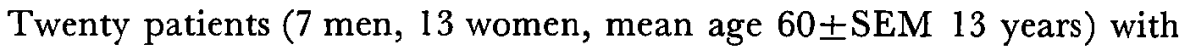
mild hypertension, defined as a diastolic blood pressure of $95 \mathrm{mmHg}$ or more (average mean blood pressure $122 \pm 60 \mathrm{mmHg}$ ), were selected from newly diagnosed, untreated patients admitted to our hospitals. None of them showed clinical, biochemical, or radiological evidence of a primary cause of hypertension. All patients were judged to have normal renal function, as evaluated from PSP tests and creatinine clearance values.

The patients were given a regular diet containing $140 \pm 10 \mathrm{mEq}$ of sodium for 14 days (" average-sodium"), followed by 5 days on a diet with $30 \pm 10$ mEq of sodium ("low-sodium "), and finally by 5 days on a diet with $280 \pm 10$ mEq of sodium (" high-sodium"). On the 4th and 5th days of low-sodium and high-sodium periods, urine was collected for analysing of sodium, potassium, and chloride levels. On the day 5, measurements of blood pressure and blood sampling were performed. The estimation of pressor doses of norepinephrine was performed early in the morning, just after sampling the 24 hour urine excretion for the day 5. 


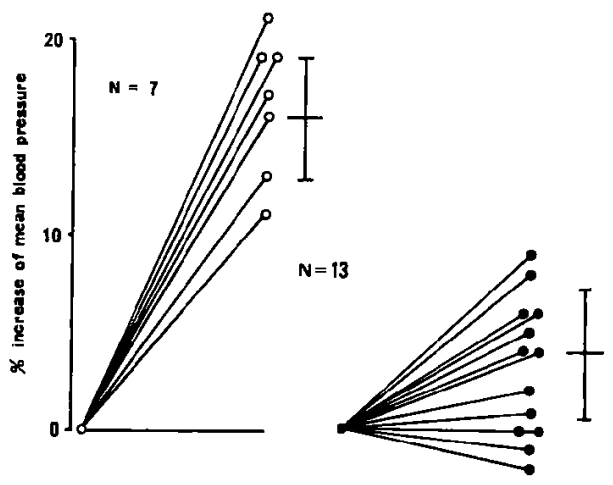

Fig. 1. Percent change of mean blood pressure in salt-sensitive (SS) and nonsalt-sensitive (NSS) patients after a change from the low-sodium to the high-sodium diet.

Blood pressure was measured with an automatic sphygmomanometer (Colin Co) every 4 hours from 07.00 to 19.00 after the patients had been in supine position for $5 \mathrm{~min}$ or longer. The average mean blood pressure was calculated from each mean blood pressure value (diastolic pressure + pulse pressure/3). The patients were classified as salt-sensitive and nonsalt-sensitive as in the previous studies. ${ }^{5), 6)}$ Patients whose average mean blood pressure value on the day 5 of the high-sodium period exceeded the value on the day 5 of the low-sodium period by at least $10 \%$ were classified as SS patients, and those whose average mean blood pressure did not change or increased less than $10 \%$ were termed NSS patients (Fig. 1).

Urinary sodium, potassium, and chloride contents were determined with an autoanalyzer. Plasma renin activity (PRA) was measured by radioimmunoassay using CIS-radioimmunoassay kits. The plasma aldosterone concentration (PAC) was measured by radioimmunoassay using Dainabot-radioimmunoassay kits.

Pressor doses of norepinephrine were estimated from the amounts of norepinephrine required to elevate mean blood pressure by $20 \mathrm{mmHg}$ above the initial level.") Norepinephrine ("Nor-adrenalin" Sankyo Co) in glucose solution $(0.01 \mathrm{mg} / \mathrm{l})$ was infused stepwise at different speeds with a perfusion pump (Turth). The infusion speed was increased when a new stable level of blood pressure had been reached. All data were expressed as mean \pm standard error of the mean (SEM), and the differences were evaluated by Student's unpaired-t or paired-t test, as appropriate. 


\section{Results}

Seven patients were classified in the SS group and 13 patients in the NSS group. The elevation ratio of mean blood pressure of the SS group was $16 \%$ and that of the NSS group was 4\%. The backgrounds of these patients are listed in Table I, which indicates no significant difference in age, blood pressure on admission and laboratory data.

The urinary sodium level on the 5th day of the high-sodium diet was significantly greater in the NSS patients than in the SS patients, though there were no significant differences in the urinary potassium level and volume. The SS patients had gained more weight than the NSS patients, but the difference was not significant. No significant change in the serum electrolyte concentration was observed (Table II).

PRA was suppressed significantly by the high-sodium diet as compared with the low-sodium diet (Fig. 2). The PAC decreased insignificantly with a

Table 1. Background of 20 Patients on Admission

\begin{tabular}{l|c|c}
\hline & Salt-sensitive & Nonsalt-sensitive \\
\hline Number & 7 & 13 \\
Age & $59.5 \pm 2.8$ & $64.4 \pm 2.8$ \\
Male : Female & $3: 4$ & $4: 9$ \\
MBP (mmHg) & $121 \pm 6.5$ & $124 \pm 5.5$ \\
S-Na (mEq/L) & $143 \pm 0.9$ & $142 \pm 1.0$ \\
S-K (mEq/L) & $4.2 \pm 0.5$ & $4.1 \pm 0.2$ \\
S-Cl (mEq/L) & $102 \pm 0.9$ & $103 \pm 1.1$ \\
S-creatinine (mg/100 ml) & $1.1 \pm 0.2$ & $1.0 \pm 0.3$
\end{tabular}

$\mathrm{MBP}=$ mean blood pressure.

Table II. Clinical and Laboratory Findings in Salt-Sensitive and Nonsalt-Sensitive Patients

\begin{tabular}{l|cc|cc}
\hline & \multicolumn{2}{|c|}{ Salt-sensitive } & \multicolumn{2}{c}{ Nonsalt-sensitive } \\
\hline Diet & low-sodium & high-sodium & low-sodium & high-sodium \\
\hline $\mathrm{S}-\mathrm{Na} \mathrm{(mEq/L)}$ & $140 \pm 2.2$ & $144 \pm 1.1$ & $140 \pm 1.0$ & $143 \pm 0.3$ \\
$\mathrm{~S}-\mathrm{K}(\mathrm{mEq} / \mathrm{L})$ & $4.5 \pm 0.5$ & $4.4 \pm 0.7$ & $4.4 \pm 0.4$ & $4.4 \pm 0.4$ \\
$\mathrm{~S}-\mathrm{Cl}(\mathrm{mEq} / \mathrm{L})$ & $101 \pm 0.9$ & $101 \pm 1.8$ & $101 \pm 1.3$ & $103 \pm 1.1$ \\
$\mathrm{U}-\mathrm{Na}(\mathrm{mEq} / \mathrm{day})$ & $56 \pm 4.9$ & $104 \pm 12.5^{*}$ & $76 \pm 13.0$ & $155 \pm 111^{*+}$ \\
$\mathrm{U}-\mathrm{K}$ (mEq/day) & $29 \pm 3.8$ & $30 \pm 3.5$ & $27 \pm 3.6$ & $32 \pm 3.9$ \\
Urine Volume (ml/day) & $1100 \pm 240$ & $1400 \pm 150$ & $1200 \pm 230$ & $1310 \pm 140$
\end{tabular}

* Statistically significant difference between low-sodium and high-sodium diet $(p<0.01)$.

+ Statistically significant difference between salt-sensitive patients (high-sodium diet) and nonsaltsensitive patients (high-sodium diet) $(p<0.01)$. 
high-sodium diet from the level under a low-sodium diet. Since the levels of PRA and PAC did not show a parallel change from a low-sodium diet to a high-sodium diet, we have employed the ratio of PAC to PRA (PAC/PRA) as a normalized index. With a high-sodium diet, the ratio PAC/PRA was significantly greater in SS patients than in NSS patients.

The pressor doses of norepinephrine in NSS patients were enhanced to a

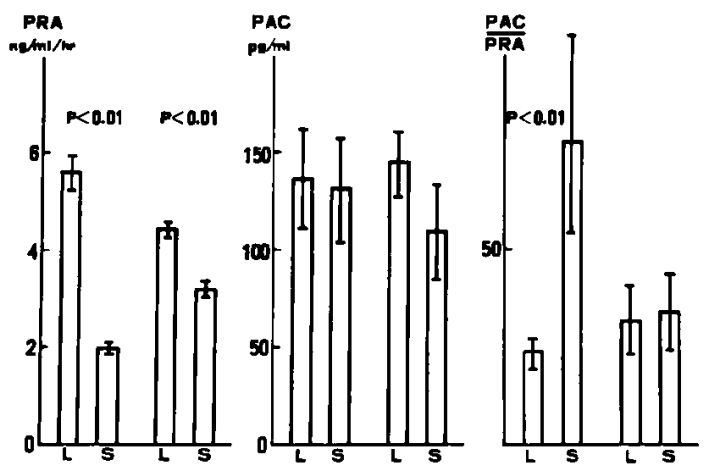

Fig. 2. Plasma renin activity (PRA) and plasma aldosterone concentration (PAC) of salt-sensitive (SS) and nonsalt-sensitive (NSS) patients given a low-sodium (L) and a high-sodium (S) diet.

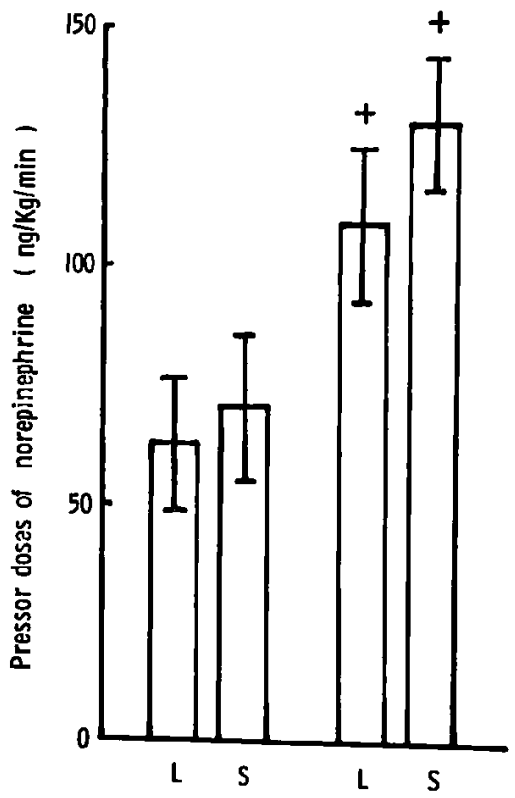

Fig. 3. Pressor doses of norepinephrine of salt-sensitive (SS) and nonsalt-sensitive (NSS) patients given a low-sodium (L) and a high-sodium (S) diet. $+: p<0.05$. Pressor doses of NSS patients were larger than those of SS patients. 
greater extent than those in SS patients. This may indicate the responsiveness to exogenous norepinephrine is enhanced more significantly in SS patients than in NSS patients. Salt intake did not affect the pressor doses of norepinephrine in either the SS or the NSS patients.

\section{Discussion}

It is speculated that the blood pressure responses to sodium-loading are variable in each hypertensive patient. Previous studies of patients with essential hypertension have attempted to classify patients on the basis of levels of PRA $^{8)}$ or plasma norepinephrine.9? Moreover hypertensive patients have been divided into $2^{5 /, 6)}$ or $3^{(0)}$ groups by the tentative criteria of response of blood pressure to salt-loading. In these trials, the patients with hypertension were subdivided into groups such as $\mathrm{SS}, \mathrm{NSS}^{5), 6)}$ or paradoxically SS groups, ${ }^{10)}$ according to response of blood pressure to salt-loading. In this study, we employed SS and NSS groups, because of the limited number of patients. Furthermore, the aim of our experimental design was to identify factors which elevate blood pressure by salt-loading.

The demonstration of 2 hypertensive groups, termed SS and NSS patients, ${ }^{51,6)}$ provides an advanced approach for the research of salt-induced hypertension. Since it was reported that the SS patients retained more sodium than the NSS patients, $\left.{ }^{5},{ }^{6}\right)$ we confirmed that the NSS patients excreted more sodium than the SS patients. However, the mechanism for sodium retention in the SS group is unclear.

Considerable attention has been focused on the role of vasoactive substances in renal natriuresis of salt-loading. However, there was no difference in the plasma aldosterone concentration in the SS and the NSS groups. As a consequence, other known factors, which are prostaglandin and dopamine, were considered. There is no evidence for an excessive release of prostaglandin or dopamine from the kidney during high sodium ingestion in the NSS patients. Fujita et al $^{6}$ ) suggested that there is an increased release of urinary $\mathrm{PGE}_{1}$ during the periods of low-sodium diet and furosemide treatment in NSS patients, and could not demonstrate the increased release of urinary PGE $_{2}$ during high-salt diet. However, since we have demonstrated an interaction between circulating prostaglandin and the pressor response to norepinephrine, ${ }^{11)}$ the attenuated pressor response to norepinephrine in the NSS patients suggests the possibility of an enhanced generation of circulating prostaglandin as compared to the SS patients. Therefore, it is suggested that the NSS patients have a higher degree of natriuresis than the SS patients, mediated by the enhanced generation of some types of prostaglandins, which in turn, dilate blood vessels 
and induce sodium excretion.

It is interesting to note that the suppression of PRA by sodium-loading was more marked in the SS group, while the PAC level did not fluctuate. Thus, there was a dissociation between plasma renin activity and the plasma aldosterone level of the SS patients during salt-loading. This suggests either that aldosterone secretion is autonomous or that regulating mechanisms other than the renin-angiotensin system are responsible for controlling aldosterone secretion. Other known regulators for aldosterone secretion are ACTH, potassium and dopamine. In combination with natriuresis, dopamine may modulate the secretion of aldosterone. ${ }^{12)}$ Increased urinary excretion of dopamine in response to sodium loading, ${ }^{13}$ reflexed the augmented dopaminergic activity of the kidney. Therefore, dopaminergic inhibition of aldosterone secretion may occur under sodium loading. From these results, it is suggested that the SS patients retained more sodium in the body as a consequence of deficient dopaminergic activity, which may diminish the inhibition of aldosterone secretion and increase the level of $\mathrm{PAC}$ in response to circulating angiotensin II.

Another factor to be considered for blood pressure elevation during sodiumloading of the SS patients is the enhanced vascular reactivity to norepinephrine. The pressor responses to norepinephrine of the SS patients were more enhanced during both sodium-deprivation and sodium-loading than those of the NSS patients. The augmentation of pressor responses to norepinephrine was one of the important factors in elevating blood pressure by sodium-loading in the SS patients. The present study provides the interesting hypothesis that the patients with an ehnanced pressor response to norepinephrine during sodium-deprivation may respond to salt-loading with an increase of blood pressure (salt-sensitive patients).

A chronic load of salt induces the elevation of peripheral vascular resistance, probably through the inhibitory effect of sodium-potassium-ATPase activity. ${ }^{14)}$ In the present study, the pressor response to norepinephrine did not increase on the 5 th day of sodium-loading. As described in the Methods, the period of sodium loading followed the sodium depletion period. Therefore, it is suggested that an insufficient sodium load masked the increase of pressor response to norepinephrine on the last day of sodium-loading in this study. We have previously observed an enhanced pressor response to norepinephrine at the sufficient load of sodium in animal experiments. ${ }^{15}$ )

Since the pressor response to norepinephrine is modulated by prostaglandins (PG), it is increased in cases with PG deficiency and decreased in cases with augmented PG. It was reported that the NSS patients excrete more urinary prostaglandin $\mathrm{E}_{2}\left(\mathrm{PGE}_{2}\right)$ during sodium depletion than the SS pa- 
tients. ${ }^{6)}$ The enhanced excretion of $\mathrm{PGE}_{2}$ in the urine of NSS patients suggests the possibility that the biosynthesis of $\mathrm{PG}$ in various tissues may be enhanced in the NSS patients. It is generally accepted that prostacyclin $\left(\mathrm{PGI}_{2}\right)$ has potent vasodilating and modulatory activity and that it may play an important role in the regulation of blood pressure. Aortic $\mathrm{PGI}_{2}$ generation is enhanced by sodium-loading and depletion. ${ }^{15}$ ) This $\mathrm{PGI}_{2}$ generation in the vascular tissue may modulate the vascular sensitivity to vasoactive substances. Therefore, we assume that there may be differences in the vascular $\mathrm{PGI}_{2}$ generating activity of the SS and the NSS patients.

In experiments employing conscious rats, with chronic catheters in the jugular vein and abdominal aorta, we found that the rats which show an enhanced pressor response to norepinephrine maintain their high vascular activity until the development of hypertension induced by DOCA/salt treatment. ${ }^{16)}$ This indicates that the enhanced pressor response to norepinephrine is probably genetic in origin.

In summary, we have divided the patients with hypertension into SS and NSS patients, according to blood pressure responses to salt-loading. The mechanisms underlying dietary sodium influences on blood pressure are not known, but it is speculated that the SS patients tend to retain more sodium and water, and have a higher pressor response to norepinephrine than NSS patients. During sodium-loading, both sodium retention and an increased pressor response to norepinephrine may contribute to the elevation of blood pressure.

\section{Agknowledgment}

We express our appreciation to Dr. Ricardo A. Faleni for reviewing the manuscript.

\section{REFERENCES}

1. Takahashi E, Sasaki N, Takeda J, Ito H: The geographic distribution of cerebral hemorrhage and hypertension in Japan. Hum Biol 29: 139, 1957

2. Murray RH, Luft FC, Block R, Weyman AE: Blood pressure responses to extremes of sodium intake in normal man. Proc Soc Exp Biol Med 159: 432, 1978

3. Tobian L: Salt hypertension. in Hypertension, ed by Genest J, Koiw E, Kuckel O, McGrawHill Book Co, New York, p 425, 1977

4. Dahl LK, Heine M, Tassinari L: Role of genetic factors in susceptibility to experimental hypertension due to chronic excess salt ingestion. Nature 194: 480, 1962

5. Kawasaki T, Delea CS, Bartter FC, Smith H: The effect of high-sodium and low-sodium intakes on blood pressure and other related variables in human subjects with idiopathic hypertension. Am J Med 64: 193, 1978

6. Fujita T, Henry WL, Bartter FC, Lake CR, Delea CS: Factors influencing blood pressure in salt-sensitive patients with hypertension. Am J Med 69: 334, 1980 
7. Distler A, Barth C, Liebau H, Vecsei P, Wolff HP: The effect of tyramine, noradrenaline, and angiotensin on the blood pressure in hypertensive patients with aldosteronism and low plasma renin. Eur J Clin Invest 1: 196, 1970

8. Brunner HR, Sealey JE, Laragh JH: Renin subgroups in essential hypertension. Further analysis of their pathophysiological and epidemiological characteristics. Circulat Res 32, 33 (Suppl I): 99, 1973

9. Dechamplain J, Vanameringen MR, Cousineau D, Marcaurele J, Yamaguchi N: The role of the sympathetic nervous system in experimental and human hypertension. Postgrad Med J 53 (Suppl 3): 15, 1977

10. Kawasaki T, Ueno M, Uezono K, Abe I, Kawano Y, Ogata M, Omae T, Fukiyama K: Salt intake and hypertension. Jpn Girculat $J$ 45: 810, 1981

11. Yoshimura M, Takahashi H, Takashina R, Kajita Y, Miyazaki T, Hachiya T, Ijichi H, Ochi $\mathrm{Y}$ : Pathophysiological role of prostaglandins in the regulation of blood pressure. in Prophylactic Approach to Hypertensive Discascs, ed by Yamori Y, Lovenberg W, Freis ED, Raven Press, New York, p 283, 1979

12. Carey RM, Thorner MO, Ortt EM: Effects of metoclopramide and bromocriptine on the renin-angiotensin-aldosterone system in man. Dopaminergic control of aldosterone. J Clin Invest 63: 727, 1979

13. Alexander RW, Gill JR Jr, Yanabe H, Lovenberg W, Haiser HR: Effect of dietary sodium and of acute saline infusion on the interrelationship between dopamine excretion and adrenergic activity in man. J Clin Invest 54: 194, 1974

14. Blaustein MP: Sodium ions, calcium ions, blood pressure regulation, and hypertension: a reassessment and a hypothesis. Am J Physiol 232: C165, 1977

15. Yoshimura M, Kaimasu I, Kitani T, Kawamura T, Takeda K, Sasaki S, Kajita Y, Nakagawa $\mathbf{M}$, Ijichi $\mathbf{H}$ : Effect of sodium loading and depletion on vascular reactivity and prostacyclin generation. Jpn Circulat J 44: 388, 1980

16. Shikuma R, Yoshimura M, Takahashi H, Takeda K, Ashizawa H, Takashina R, Inoue A, Ochi Y, Ijichi $H$ : The continuous direct monitoring of blood pressure in the conscious rats, and aberration of pressure response with salt loading. Jpn J Hypertension 4: 69, 1981 